\title{
The Science Behind G Protein-Coupled Receptors (GPCRs) and Their Accurate Visual Representation in Scientific Research
}

\author{
Amy C. Sojka, Kevin M. Brennan, Evelyn T. Maizels, and Christine D. Young
}

G Protein-Coupled Receptors (GPCRs) are transmembrane (TM) proteins that span the cell membrane seven times, and contain intracellular and extracellular domains, comprised of connecting loops, as well as terminal extension sequences. GPCRs bind ligands within their transmembrane and/or extracellular domains. Ligand binding elicits conformational changes that initiate downstream intracellular signaling events through arrestins and G proteins. GPCRs play central roles in many physiological processes, from sensory to neurological, cardiovascular, endocrine, and reproductive functions. This paper strives to provide an entry point to current GPCR science, and to identify visual approaches to communicate select aspects of GPCR structure and function with clarity and accuracy. The overall GPCR structure, primary sequence and the implications of sequence for membrane topology, ligand binding and helical rearrangements accompanying activation are considered and discussed in the context of visualization strategies, including two-dimensional topological diagrams, three-dimensional representations, and common errors that arise from these representations.

\section{OPEN ACCESS}

\section{Introduction}

G Protein-Coupled Receptors (GPCRs) are transmembrane (TM) proteins that span the cell membrane seven times, and contain intracellular and extracellular domains comprised of connecting loops as well as terminal extension sequences. GPCRs bind ligands within their transmembrane and/or extracellular domains. Ligand binding elicits conformational changes that initiate downstream intracellular signaling events through arrestins and $G$ proteins (Figure 1; Katritch et al., 2013). GPCRs play central roles in many physiological processes from sensory to neurological, cardiovascular, endocrine, and reproductive functions. GPCRs represent one of the largest gene families in the human genome, encoding approximately 800 unique proteins (Fredriksson et al., 2003). GPCRs' unique structure and cell surface location make them ideal targets for various drug therapies, assuring interest from the pharmaceutical and clinical medicine communities (Vischer et al., 2011). It is estimated that roughly $40 \%$ of the pharmaceuticals currently marketed, target GPCRs (Vischer et al., 2011).

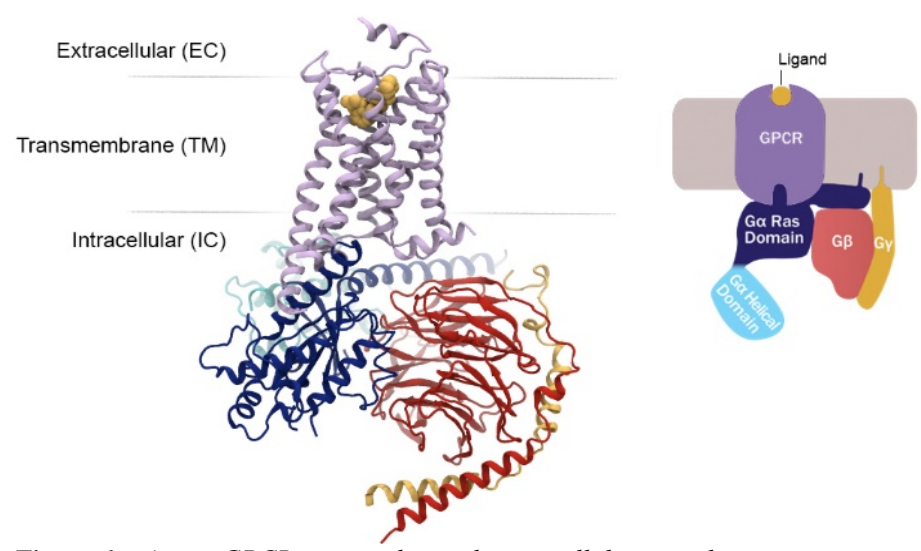

Figure 1. Active GPCR in complex with intracellular signaling partner protein the heterotrimeric G protein Gs. (PDB 3SN6, human $\beta 2$-adrenergic receptor with bound ligand BI-167107, bovine Gs; Rasmussen et al., 2011) This image was made with Visual Molecular Dynamics (VMD). VMD is developed with NIH support by the Theoretical and Computational Biophysics group at the Beckman Institute, University of Illinois at Urbana-Champaign (Humphreys et al., 1996).

The elucidation of x-ray crystallographic structures of GPCRs has been monumental to the research in understanding the function and conformational flexibility of GPCRs (Costanzi 2014; Venkatakrishnan et al., 2014). Understanding how ligand binding alters the structure and function of GPCRs to mediate signaling has undergone an expansion in recent years (Katritch et al., 2013). Concepts such as ligands acting as functionally selective biased agonists to elicit a specific subset of signaling responses are now at the forefront of GPCR research (Andresen 2011). Development of allosteric ligands extends the repertoire of GPCR regulators (Smith 2010). GPCRs were originally considered to be monomeric, but increasing evidence indicates that they can form dimers and oligomers as well (Ferre et al., 2014).

Accurate representations of GPCRs aid in the communication of emerging insights into the dynamic relationships between structural 
and functional features of GPCRs. The availability of more than 120 structures, covering 32 unique GPCRs (Piscitelli et al., 2015), has been described as a "crystallization boom" (Costanzi 2014), with rapid growth emerging due to recent technological advances in both crystallization and structure detection. The availability of growing numbers of experimentally-determined GPCR structures, as well as improved homology-modeling of unknown target proteins based on a wider field of experimentally determined GPCR template structures, allows visual representation of GPCRs to be built upon the basis of known or modeled three-dimensional structures. Nevertheless, several challenges to accurate communication of GPCR structure remain.

The goal of this paper is to provide strategies to address common visual representation challenges for GPCRs, and to identify errors that may arise from an incomplete understanding of GPCR structure. GPCRs comprise a subclass of the alpha-helical membrane protein class and many of the challenges and solutions would also be applicable to other members of this class, such as ion channels and transporter proteins. This paper focuses on GPCRs specifically, as the subject matter for several major reasons: 1) prominence of GPCRs in current scientific research endeavors; 2) prevalence of GPCRs as pharmaceutical targets; and 3) indication of confusion translating two-dimensional topology diagrams into three-dimensional environments, a confusion resulting in errors encountered with some regularity in GPCR representations in the literature, but not in representations of other membrane proteins. Errors incorporated into the scientific literature have made their way into the educational literature, harkening Goodsell and Johnson's warning that visual communicators should, "avoid polluting the literature (both scientific and popular) with deceptive imagery. This is particularly important for scientists, since pictorial errors in primary scientific publication, which are often persistent sources of information, may be propagated for decades in educational and outreach publications." (Goodsell 2007).

\section{Protein Representation}

Visual communication of macromolecular structure, including protein structure, has a rich history that has been reviewed in several comprehensive texts including: Mura et al. [supplement], 2010; Perkins, 2005a; Perkins, 2005b; and Richardson et al., 1992.

A milestone in the depiction of protein structure was the development of the ribbon mode of representation, as popularized by Jane Richardson in her transformative hand drawn illustrations (Richardson et al., 1992). The Richardson ribbon representation mode stylizes and abstracts the alpha-carbon trace of the peptide backbone into recognizable secondary structure motifs (Figure 1, left panel; see also Figures 2, 4, 8, 9 and 11 and Table 1). Side chain atoms are excluded from the representation. Helices are depicted as simplified spirals approximating the alpha-carbon tracing, and beta strands are represented as flattened arrows. The ribbon representation mode remains the most frequent method of depiction of structure in scientist-to-scientist peer communication (Mura et al., 2010; Goodsell 2005). Richardson also developed a related cartoon mode using rods instead of spirals to represent helices, a depiction which further simplifies and abstracts protein positional information into recognizable motifs (Figure 2, middle panel; see also Figures 10 and 12 and Table 1.). Additional alpha carbon-based representation modes include trace, narrow ribbon, and tube representations.

\begin{tabular}{|l|l|l|l|}
\hline $\begin{array}{l}\text { Representation } \\
\text { Mode }\end{array}$ & \multicolumn{1}{|c|}{ VMD } & \multicolumn{1}{|c|}{ PyMOL } & \multicolumn{1}{c|}{ Chimera } \\
\hline Ribbon & NewCartoon & Ribbon Cartoon & Ribbon \\
\hline Rod-Cartoon & Cartoon & $\begin{array}{l}\text { Cylinder } \\
\text { Cartoon }\end{array}$ & PipesAndPlanks \\
\hline Stick & Licorice & Stick & Stick \\
\hline Ball and Stick & CPK & Ball and Stick & Ball-and-stick \\
\hline $\begin{array}{l}\text { Space-filling } \\
\text { Sphere }\end{array}$ & VDW & Sphere & Sphere \\
\hline Surface & Surf/QuickSurf & Surface & Solid surface \\
\hline
\end{tabular}

Table 1. Terminology for representation modes within three commonly-used software packages: Visual Molecular Dynamics [VMD] (Humphreys et al., 1996); UCSF Chimera (Peterson et al., 2004); and Pymol (The PyMOL Molecular Graphics System, Schrödinger, LLC; http://pymol.org/)(Mura et al., 2010).

Advantages of the various alpha-carbon based representation modes are afforded by the visibility of interior elements, allowing the viewer to easily grasp relationships that contribute to the overall fold of a protein (Harle 2012a; O'Donoghue et al., 2010). The ribbon and rodcartoon modes both clarify the contributions of secondary structure motifs. The further simplification of helical shapes into rod segments in rod-cartoon mode allows the viewer to distinguish the relative positions of multiple helices in a crowded representation, for example when two or more structures of related proteins are superimposed (See Figure 12; Standfuss et al., 2011, Figure 2a; and Park et al., 2012, Figure 3a).

A limitation of the various alpha-carbon trace-based representation modes including ribbon and rod-cartoon, derives from the method of abstraction that depicts only peptide backbone atoms. It is not possible to show the contribution of side chain atoms to the structure and function of the protein in any of these representation modes. An additional limitation of the rod-cartoon mode is that it presupposes that helices can be adequately represented as straight rod segments. Straight rod segments suggest rigidity of helices and would underestimate the contributions of flexibility, kinks and bends to the position and motion of helices (Figure 2). This limitation merits consideration in choosing a representation mode, depending on the communication goals of the illustration, since flexibility and helical kinks are well-described features of GPCR TM helices (Katritch et al., 2013; van der Kant 2014).
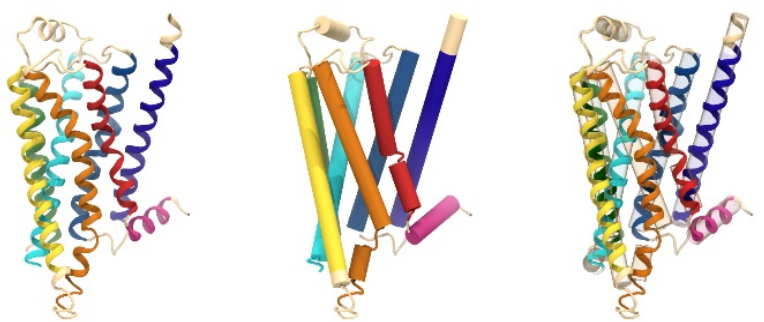

Figure 2. Ribbon representation mode (left), rod-cartoon representation mode (middle) and comparison of ribbon and rod representations (right) colored 
according to helical segment,TM1-7 and H8, rainbow sequence ranging from indigo/blue (N-terminus) to red/magenta (C-terminus). Both modes make the positions of alpha helices within the overall fold of the GPCR readily visible. A limitation of the rod-cartoon mode is that representation of helices as series of straight segments may underestimate the contribution of bending to helix shape. A curved shape is apparent in the ribbon representation of transmembrane helix 6 (TM6; orange), but not in the rod representation. [turkey $\beta 1$-adrenergic receptor, $P D B 2 Y C W]$. Image created in $V M D$.

Other representation modes depict the contribution of all atoms (excluding hydrogen) to a protein structure, and fall into two main categories. The first category makes covalent bonds explicit, and includes stick, and ball-and-stick representations (Goodsell 2005, Table 1). The second category makes the volumes occupied by atoms explicit, and comprises the space-filling "sphere" representation mode, using spheres calculated to represent contact distances (van der Waals radii) between atoms (Goodsell 2005, Table 1). One advantage of these modes is the fuller representation of atoms, especially side-chain atoms. A main disadvantage is that the overall structure of a protein or region of protein may be hard to understand because structural folds and secondary structural elements cannot be seen due to obscuring atomic details (Harle 2012a; Richardson et al., 1992). These all-atom representation modes are also useful for representation of small molecules such as ligands (Figure 3), since molecules that are not peptides cannot be visualized by the alpha-carbon trace methods.

Protein representations that combine two or more modes may provide advantages for the viewer. A stick representation mode, providing detailed amino acid side chain information for a few selected residues, can be superimposed over a backbonerepresentation, such as ribbon representation mode. This combination representation provides the advantages of abstraction of the simplified trace representation for comprehension of overall structure, while presenting full atomic detail specifically for those side chain atoms that are most relevant to the concept that the illustration is communicating (for example, see Figure 11).

A final group of representation modes for protein structure comprises surface representation modes (Table 1). Surface modes are useful to visualize the shapes of cavities such as ligand binding sites and channels. A recent development is the use of a crosssectional cut in combination with a surface representation to depict the shape and occupancy of ligand-binding cavities, allowing the viewer to see buried portions of the surface that would otherwise be occluded from view (Figure 3; see also additional examples: Manglik et al., 2012, Figure 2a,b, and Burg et al., 2015, Figure 2.

The surface representations also can be useful to depict contribution of surface electrostatic potentials or hydrophobicity to forming protein interaction surfaces (O’Donoghue et al., 2010). While any of the protein representation modes may be used to show hydrophobicity or electrostatic potential by encoding the additional information through use of color, surface representation is a frequent choice for this kind of visualization. For example, Krumm et al. (2015) use surface representation, enhanced by use of color encoded to depict electrostatic potential, to show charge distribution across ligand binding surfaces of GPCRs (Krumm et al., 2015, Figure 2). As another example, Figure 7 shows a surface representation of $\beta 1$ adrenergic receptor, with color encoded to depict hydrophobicity of local residue side chains, red for hydrophobic and blue for hydrophilic residues.

Current guidelines for protein representation have been the subject of several recent reviews that will serve the medical illustration community well. Papers by O’Donoghue et al. (2010), Mura et al. (2010), and Johnson and Hertig (2014) serve as practical guides to the visualization of protein structures, reviewing different modes of representation, guidelines as to appropriate level of detail and complexity, and software to produce accurate illustrations. There are several papers that expound upon the importance of choosing a certain representation to match the communication goal of the illustration. Johnson and Hertig (2014) in particular analyze different illustration audiences, discussing visualization considerations for the scientific, education, and outreach audiences. Articles in the cell biology and biochemistry teaching literature discuss the choice of representations for education audiences (Harle 2012a; Harle 2012b; Jenkinson 2013; Loertscher et al., 2014; Dahmani et al., 2009). Kramer et al. (2012) identifies the schematic, schematic-realistic, and realistic styles of illustration and the benefits of using each style.
A.
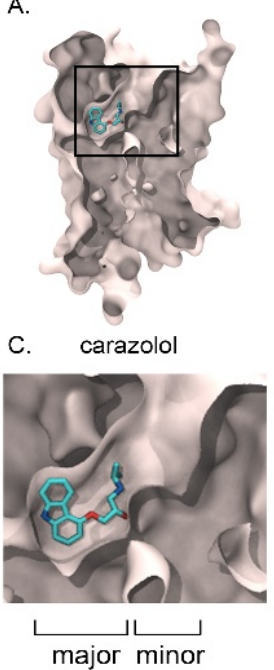

B.
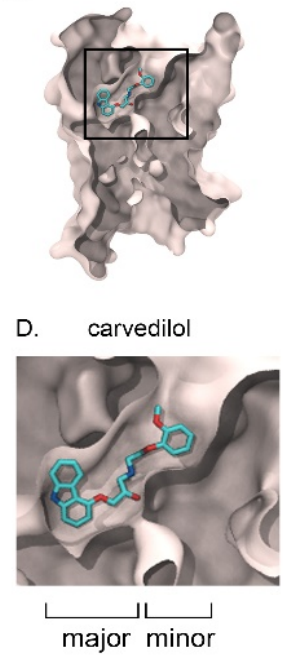

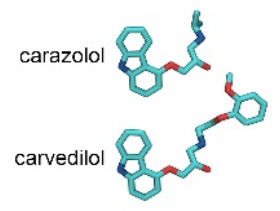

E. no ligand shown

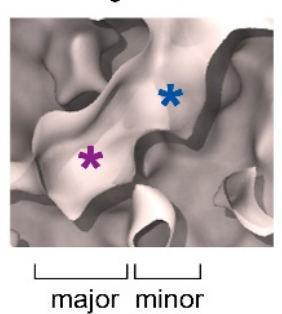

Figure 3. Cross sectional cut in combination with surface representation (VMD QuickSurf) reveals relationships between ligand and binding cavity buried within the transmembrane domain. Ligands shown in stick (VMD licorice) mode. Turkey $B 1$-adrenergic receptor B1AR with bound carazolol [PDB 2YCW; panels $A, C]$; $B 1 A R$ with carvedilol $[P D B 4 A M J$; panels $B, D] ; B 1 A R$ with ligand hidden $[P D B$ $2 Y C W$; panel E]. Panel $A$ (whole) and $C$ (inset): the smaller ligand carazolol occupies a portion of the available binding cavity, corresponding to the major pocket (compare with area marked with purple asterisk in panel E). Panel B (whole) and D (inset): The larger ligand carvedilol occupies both the major pocket (purple asterisk) and minor pocket (area marked with blue asterisk in panel E). Further details on the ligand binding cavities and major and minor pockets are given in text section on ligand binding, below.

The Nature Methods 'Points of View' section, authored by medical illustrator Bang Wong, stresses the importance of simplicity in representation and the concept of salience which allows a viewer to 
focus on the most relevant parts of an illustration (Wong 2010; 2011b). The concept of salience needs to be considered when designing an illustration to meet communication goals. As Wong (2010) indicates, "Salience is a visual quality that sets an object apart from its surroundings." Salience is visual emphasis provided to elements in a visual field by employing contrast in features that our visual systems readily detect, such as hue, value, saturation, mark size, and mark direction. For example, saturated colors will pop out against a field of muted colors, dark colors will pop out among a field of lighter tones against a light background, or large or bold text marks against a field of smaller marks. Salient visual features should be used for those elements of a composition that correspond to the most relevant information (Wong 2011a). The key communication goal of the illustration should be presented in a highly salient manner, allowing the viewer to quickly comprehend important information with a minimum amount of distraction. Secondary or supporting information should be presented in a low salience manner (Wong 2010). An example of an illustration making use of the concept of salience can be seen in Figure 11, where the saturated orange and cyan hues attract the viewer's eye to the important residues and the helices in which these residues are found. Other helices not relevant to the main communication goal of the illustration are muted and lighter in value, closer to the value of the white background to avoid distraction.

\section{GPCR Structure Overview}

The overall GPCR structure is composed of a transmembrane domain, an intracellular domain, and an extracellular domain (Figure 4). The transmembrane domain is comprised of the characteristic seven helical spans traversing the membrane in an anti-parallel fashion, arranged into a roughly cylindrical bundle. The transmembrane domain is highly conserved: all members of the GPCR superfamily share the iconic fold comprised of the seven transmembrane helices (TM1-TM7) (Katritch et al., 2012).

The intracellular domain is comprised of three loops joining the helices at the intracellular face, together with C-terminal extension sequences (Millar 2010). An eighth helix (H8), which does not cross the membrane, but rather lies parallel to the membrane at the intracellular surface of the lipid bilayer, is a feature of the $\mathrm{C}$-terminal extension sequence region of most GPCRs. The intracellular domain extends into the cytoplasmic space of the cell. Intracellular domains also tend to be conserved in size and shape in order to bind to and interact with a common set of signaling partners (Katritch et al., 2012).

The extracellular domain is comprised of three loops joining the helices at the extracellular face, together with N-terminal extension sequences. The extracellular domain extends into the extracellular space, and allows for communication with soluble ligands. The extracellular domains display the least degree of conservation, varying widely in size and shape (Katritch et al., 2012). Most class A rhodopsin-like receptors have relatively small extracellular domains with minimal N-terminal extensions. By contrast, long $\mathrm{N}$-terminal extensions characterize the class $\mathrm{C}$ glutamate-like receptors, the class B secretin-glucagon-like receptors, and a small subset of the class A rhodopsin-like receptors (Venkatakrishnan et al., 2014).

Visualization considerations for the depiction of GPCR structures include choice of vantage and representation mode. The side view (terminology adopted from Katritch et al., 2013), viewing the GPCR from within the plane of the membrane, is frequently used to depict the overall structure of the GPCR in scientific literature. The side view affords the ability to see all domains in relation to each other, and can provide context in depicting the relationship of the GPCR to the membrane and cellular compartments. A disadvantage of the side view is that some helical details may be obscured by more proximally located intervening helices. The side view can be supplemented by additional views, including intracellular and extracellular views (Katritch et al., 2013), which allow appreciation of local helical arrangements. The advantages of the intracellular and extracellular views are the increased visibility of intracellular or extracellular portions of all helices respectively, however, distal details are hidden in these views, and it can be difficult to grasp relationships between the different segments of the protein. Moreover, relation to the membrane and to overall cellular context is not provided by either of these views. The Richardson ribbon representation is useful for a first overview of a structure (O'Donoghue et al., 2010) and is the most commonly used representation mode found in structural reports (Goodsell 2005). (For examples, see Manglik et al., 2012, Figure 1, [panel a: side view left; extracellular view middle top; intracellular view middle bottom] and side view, Katritch et al., 2013, Figure 1).

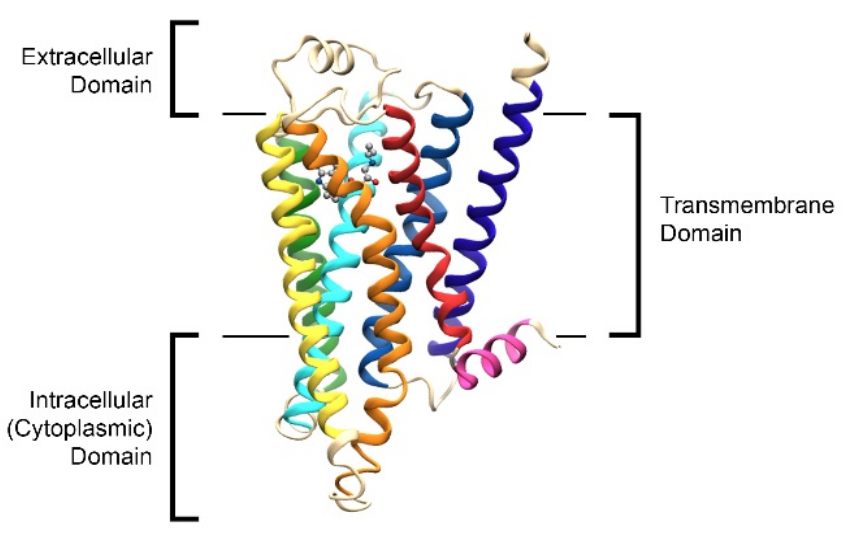

Figure 4. GPCRs are made up of extracellular, transmembrane, and Intracellular domains. [B1AR with bound ligand carazolol, PDB ID $2 Y C W]$. Side view, lines indicate membrane hydrophobic core boundaries. Image created in VMD. 


\section{GPCR Primary Sequence and Membrane Topology}

GPCRs are single peptide chains that are folded into a "bundle of rods" cylindrical shape. The primary sequence describes the single polypeptide chain by identifying the individual amino acid residues of the protein from $\mathrm{N}$ Terminus to C-Terminus. Determination in the 1980's of the primary sequences of rhodopsin and the $B$ adrenergic receptors (Hargrave et al., 1983; Dixon et al., 1986; Kobilka et al., 1987), first prototypes of the GPCR superfamily, were scientific breakthroughs that provided early insight into the relationship of these proteins to the membrane. Inspection of these sequences revealed the presence in each of seven sequence spans of highly hydrophobic residues. The seven hydrophobic sequence spans were recognized to correspond to transmembrane alpha helices, and intervening hydrophilic segments corresponding to connecting loops. Thus the single polypeptide chain was understood to be folded into a structure with anti-parallel helices comprising the transmembrane domain and connecting loops defining the intracellular and extracellular domains, respectively.

The directionality of the transmembrane helices as they pass through the membrane, and the disposition of intervening connecting loops as "inner" or "outer" in relation to the membrane, are described by the term "membrane topology". The concept of membrane topology is the subject of a comprehensive review (von Heijne 2006). Von Heijne defines the term within the glossary as, "a specification of the number of transmembrane helices and their in and/or out orientations across the membrane in a membrane protein." Von Heinje notes that, "In the world of membrane proteins, topology defines an important halfway house between the amino-acid sequence and the fully folded three-dimensional structure." A lack of differentiation by artists and/or viewing audience members between what is understood as membrane topology, at essence a two-dimensional concept, and the fully folded three-dimensional shape of a protein, lies behind several misconceptions and misrepresentation of the structure of GPCRs (discussed below).

The helical spans and intervening loops of a GPCR can be mapped graphically as a snake plot. The snake plot is a specialized diagram that takes the information of the primary sequence and arranges it into its appropriate helices and/or loops from N-Terminus to C-Terminus (Lefkowitz 2013). The snake plot incorporates spatial information in a two-dimensional manner by defining the topological relationship of the protein to the membrane, indicating what portions of the GPCR are transmembrane, intracellular, and extracellular. No three-dimensional spatial information is implied by the snake plot. Scientists understood the meaning of the snake plot as a representation of membrane topology of a GPCR decades before actual threedimensional structural data for GPCRs became available
(Palczewski et al., 2000; Hargrave et al., 1983; Kobilka et al., 1987, see Figure 3; Palczewski et al., 2000). The snake plot continues to be an important mode of representation because it allows insight into the role of specific amino acid residues within the context of their respective helices. Within the snake plot, all amino acid residues are visible; nothing is occluded, which provides an advantage in contexts where three-dimensional representations might suffer from occluded detail. The snake plot identifies specific residues, such as those that define ligand binding pockets or contribute to receptor activation, residues that participate in interhelical or helix-to-loop interactions such as ionic locks or disulfide bonds, residues subject to post-translational modifications, as well as conserved positions, disease-associated mutations, or engineered mutations (see Langer 2012, Figure 2; and Shibata et al., 2009, Figure 3B) Snake plots can be readily generated for GPCRs of interest by use of the GPCRDB (GPCR database) web-based tools (http://tools.gpcr.org/; (Isberg et al., 2014).

The snake icon was derived from the snake plot keeping the $\mathrm{N}$-terminus to $\mathrm{C}$-terminus mapping. Viewers have recognized the seven parallel rods as an iconic representation of the transmembrane helices and the snake icon has been adopted as a frequent schematic representation of the GPCR (Couty et al., 2005, Figure 1).

The two-dimensional snake icon topology diagram correctly forms into a shape with three dimensions via "wrapping" or "rolling" (Figure 5). The GPCR overall structure is roughly cylindrical with TM1 lined up next to TM7, with helices arrayed in numerical order in a counter-clockwise manner when viewed from the extracellular view (Lee et al., 2015). This is referred to as the "bundle of rods" that are recognized as the GPCR's three-dimensional structure. 
$2 \mathrm{D}$ representation $=$ unwrapped cylinder
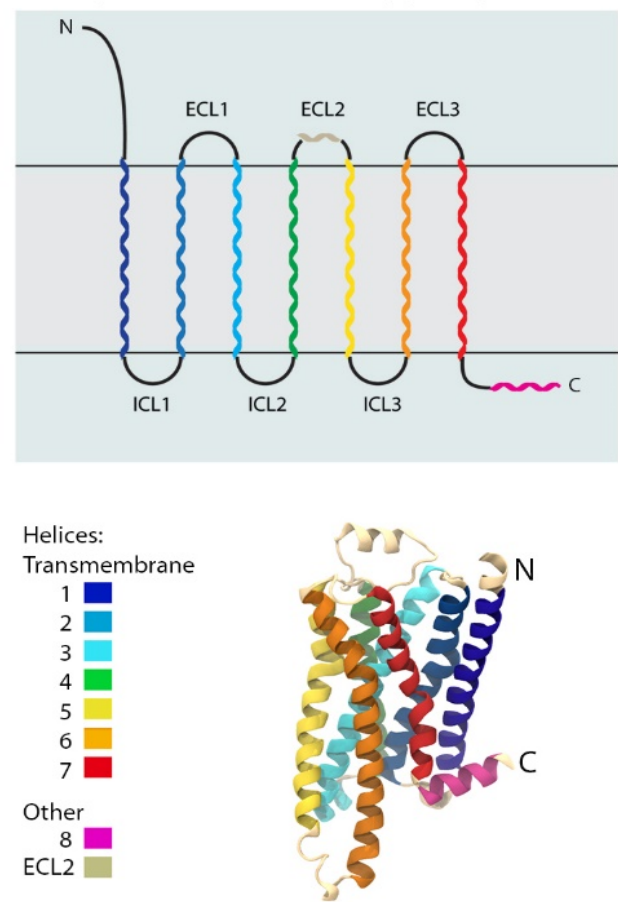

Figure 5. Accurate Translation of two-dimensional topology to threedimensional cylindrical shape. Structure depicted is Turkey $\beta 1$ Adrenergic Receptor, $P D B 2 Y 00$, image rendered in VMD.

\section{Schematic Misrepresentations Arising from Snake Plot Topology Diagrams}

The snake plot and snake icon topology diagrams only depict two-dimensional information and a common misrepresentation of GPCRs is to use a representation of a snake icon that has applied a false representation of depth, presenting the three-dimensional snake as an element within in a three-dimensional environment. Such a visual representation ignores the actual three-dimensional structure of the GPCR. Notably, the pseudo-three-dimensional snake has no interior core, so representation of concepts such as ligand binding become problematic. A particularly prevalent example of a pseudo-three-dimensional snake image is found on the cover of the twelfth edition of Goodman and Gilman's, "The Pharmacological Basis of Therapeutics" (McGraw Hill 2011), a widely used medical student- and graduate-level pharmacology text (visit publisher; stock image source Medimation). This same stock image also appears on the cover of the book entitled "G Protein-Coupled Receptors: From Structure to Function," editors Giraldo and Pin (Royal Society of Chemistry 2011; visit publisher). The image also forms the cover of the June 2014 issue of Trends in Pharmacological Sciences, a scientific review journal published by Cell Press, to serve as visual indicator of a special review series on ligand bias in GPCRs co-edited by Robert Lefkowitz, the 2012 Nobel
Laureate in Chemistry, (Schaffhausen 2014; link). Further examples of pseudo three-dimensional snake images are found in the scientific literature (Daaka et al., 2012 Figure 1; Innamorati et al., 2011, Figure 2).

A second common mistake has evolved from the snake icon topology diagram. This representation attempts to merge the mapping information from the snake icon with the understanding of the GPCR as a "bundle of rods" without understanding the overall cylindrical arrangement of the helices in the bundle. This is a representation that takes the snake plot helices and re-arranges them in a staggered manner, front-to-back, to give the GPCR helices a zig-zag or accordion arrangement (Figure 6).

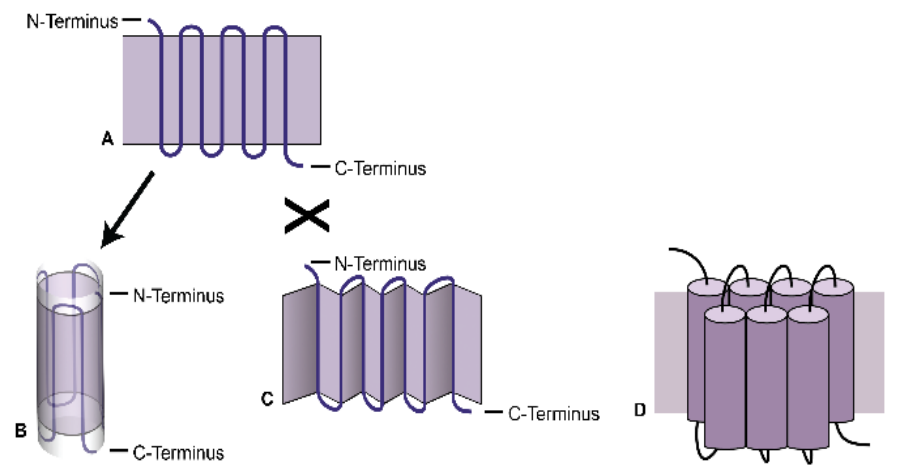

Figure 6. Two-dimensional topology (A) to three-dimensional shape is correctly related by rolling (B), but leads to incorrect shape by folding or staggering (c). Erroneous folding/staggering places helices in a zigzag or accordion arrangement $(D)$.

This accordion is a misrepresentation of spatial relationships between GPCR helices (see examples: Sodhi et al., 2004, Figure 5; Alvarez et al., 2013 Figure 4; Goddard et al., 2012, Figure 1; Siu 2010, Figure 2).

Such erroneous representations as the pseudo-threedimensional snake and accordion representations prevent the viewer from building correct mental concepts of how helices interact with each other, how helices interface with the membrane phospholipids, how ligands might bind within the receptor structure and how conformational changes might open a docking site on the intracellular face to communicate with intracellular signaling partners, as discussed below.

\section{GPCR Relationship to the Membrane}

Many of the residues that form the GPCR transmembrane helices are hydrophobic, allowing the transmembrane domain to reside embedded within the hydrophobic portion of the lipid bilayer. The intracellular and extracellular loops tend to be more hydrophilic, permitting these structures to reside in contact with the cytoplasmic and extracellular aqueous environments (Figure 7). 


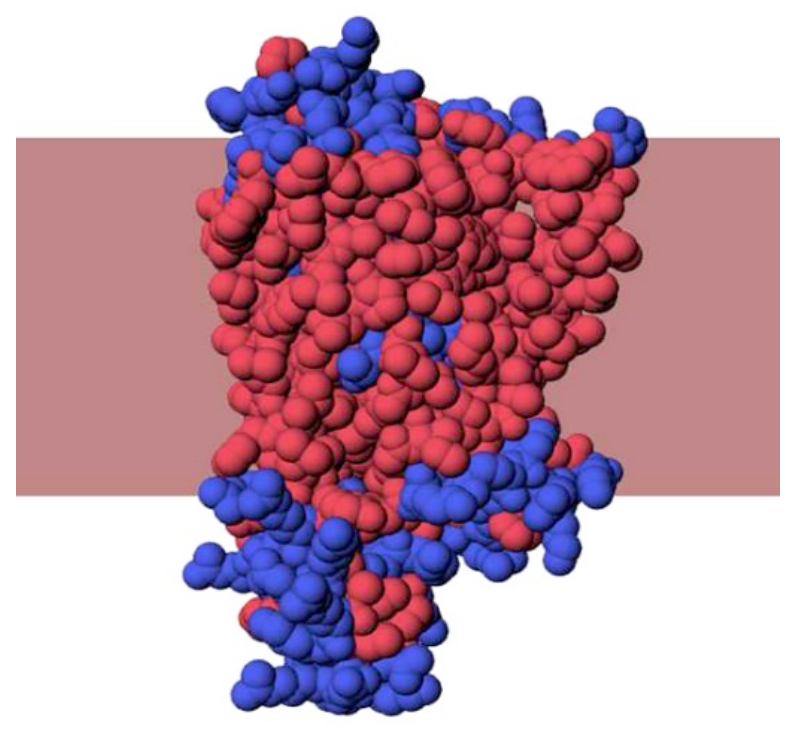

Figure 7. Hydrophobicity of a Turkey $\beta 1$-Adrenergic Receptor (PDB ID 2YCZ). Red indicates hydrophobic residues and blue indicates hydrophilic residues. Image from jmol via RSCB PDB website, surface representation.

Recognizing that the hydrophobic helical spans traverse the membrane roughly parallel to each other and perpendicular to the plane of the membrane helps to ensure the size relation of the GPCR, which is vital to correctly illustrate function. Helices five (TM5) and six (TM6), notably, have intracellular extensions. The Orientation of Proteins in Membranes Database (OPM) (Lomize et al., 2012) is an excellent resource to reliably guide size relationship depictions of membrane proteins including GPCRs. (http://opm.phar.umich.edu/). The OPM database calculates and catalogs the orientation and position of membrane proteins with respect to the phospholipid bilayer. OPM displays membrane proteins with boundary markers within static images and three-dimensional interactive windows, and provides downloadable PDB coordinate files for use within molecular visualization programs. Two sets of markers denote the locations of inner (blue) and outer (red) boundaries of the hydrophobic core of the phospholipid bilayer respectively (Figure 8). Note that the provided markers indicate the boundaries between the hydrophobic tail and hydrophilic headgroup regions of the associated phospholipids, rather than representing the entire length of the associated phospholipids.

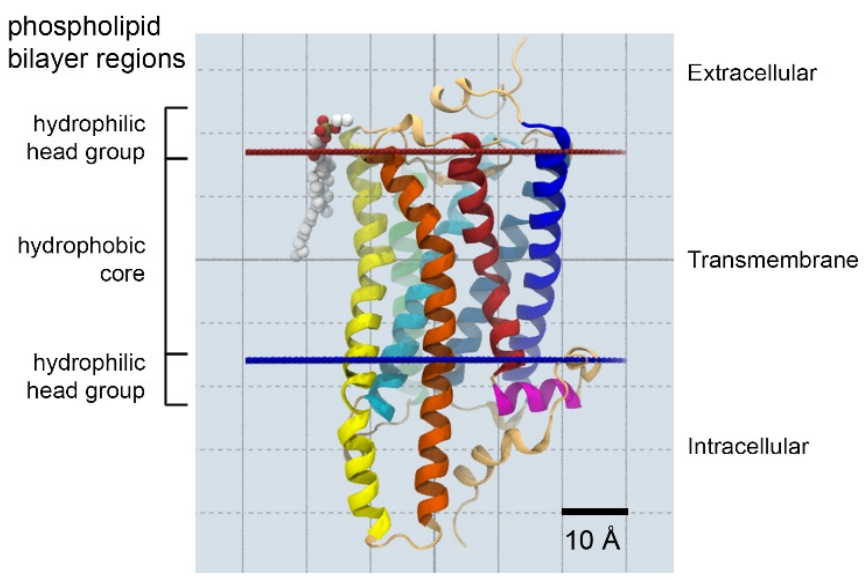

Figure 8. Size relationships of squid rhodopsin with relation to the membrane (side view). Image shows squid rhodopsin [PDB 2Z73] with entrapped cocrystallized membrane phospholipid molecule, phosphatidylcholine (spheres). Size relationships based on co-crystallized phosphatidylcholine (left labels) are compared to the membrane hydrophobic core as denoted by red (extracellular) and blue (intracellular) boundary marker coordinates provided by OPM. The 10 A grid was generated in VMD as a function of the ruler tool (orthographic viewport).

With respect to relation of GPCRs to the membrane, an issue that engenders misrepresentation is the positioning of phospholipid molecules in the interior of GPCRs, such as between helices. Johnson and Hertig (2014) note that some viewers misunderstand the meaning of the helical ribbon representation, noting, "sparse ribbon ... is often mistaken for a physical depiction of a protein". Villifane and colleagues (2011), biochemisty education researchers, document a persistent misapprehension of the helical ribbon representations. That misconception is that the side-chains of helices would be positioned pointing inward, filling the interior of the ribbon, rather than facing outward, and thus the ribbon/rod would be mistakenly understood to represent the full volume of the helix. This understanding erroneously suggests that there might be spaces between helices. It is a simple, but incorrect leap to deduce that phospholipids might be intercalated into those spaces (see examples: Guervich \& Gurevich 2006, Figure 2; Sodhi et al., 2004, Figure 5; Alvarez et al., 2013, Figure 4).

\section{Ligand Binding}

Both endogenous, or naturally occurring, and pharmacological ligands induce a variety of responses within the cell. Ligand binding causes conformational changes which facilitate interactions with binding partners to alter downstream signaling.

Ligand binding can be represented visually in the context of the whole GPCR, or alternately as a feature of a smaller region or domains within the GPCR. Visualization choices include decisions about the view from which to depict features and about the representation mode(s) to use. 


\section{Ligand Binding: Side View}

The side view can be useful to depict the location of ligand binding site within the overall GPCR structure. Side views allow an understanding of the location of ligand binding sites with respect to depth within the structure. Smaller ligands bind within ligand binding cavities embedded within the outer portion of the transmembrane bundle (Figure 9).
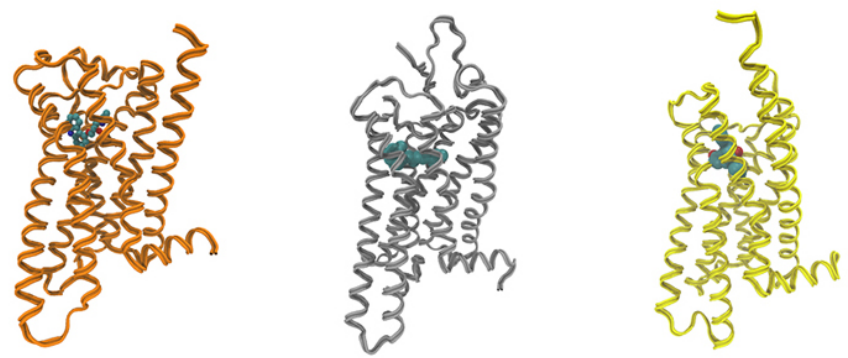

Figure 9. Ligand binding sites of $\beta 2$-Adrenergic Receptor with bound carazolol (PDB ID 4GBR, human); rhodopsin with bound all-trans retinal (PDB ID 3PQR, bovine metarodopisn II); and delta opioid receptor with bound naltrindole (PDB ID 4N6H, human). Image created in VMD.

In contrast, larger ligands are accommodated more superficially, within ligand binding sites formed by extracellular loops and N-terminal extension sequences (Lee et al., 2015). Ribbon, rod-cartoon, or other alpha-carbon trace-based three-dimensional representation modes can allow the viewer to see into the interior of the overall structure without intervening or obscuring features. An additional representation mode useful for the side view is the combination of surface with cutaway cross section, which facilitates understanding details of the shape of the ligand and ligand binding sites (see Figure 3 ).

\section{Ligand Binding: Extracellular View}

The extracellular views can also be useful to depict the location of ligand binding sites within the overall GPCR structure, showing the location and relationships of ligands to binding sites in a different way (Figure 10). The GPCR helices divide the interior ligand binding cavity into two pockets; designated as major and minor binding pockets, due to the diagonal angle of TM3. The major and minor pockets are visualized effectively from the extracellular view, where contribution of individual helices to each pocket can be shown (Figure 10). The major binding pocket, proposed as the site where ligands that affect $G$ proteins bind, is formed primarily by helices 3,4,5 and 6 . The minor binding pocket, proposed as the site where ligands that affect arrestins bind, is formed primarily by helices 1,2,3 and 7 (Rosenkilde et al., 2010). This can be seen in Figure 10, panel $\mathrm{C}$ with the major binding pocket depicted in purple, and the minor binding pocket represented in blue. In the case of the $\beta 1$ adrenergic receptor, as shown in Figure 10, the ligand carazolol (Moukhametzianov et al., 2011), bound entirely within the major pocket (panel A) alters signal exclusively to the $G$ protein pathways, without influence on arrestin pathways. By contrast the larger ligand carvedilol, bound in both major and minor pockets of $\beta 1$ adrenergic receptor (Figure 10, panel B) affects both $\mathrm{G}$ protein signaling and arrestin signaling (Warne et al., 2012).
A

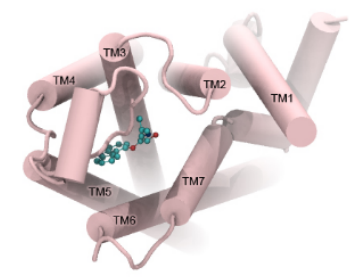

B

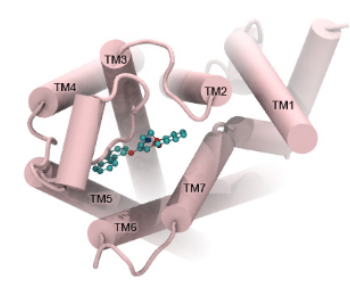

C

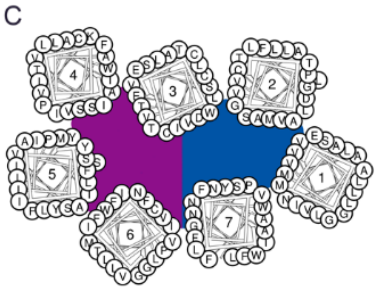

Figure 10. Extracellular view of A: Turkey $\beta 1$-Adrenergic Receptor B1AR with carazolol as bound ligand $[P D B 2 Y C W]$ in the major binding pocket, B: BIAR with carvedilol as bound ligand [PDB 4AMJ] extending into the minor binding pocket. $A$ and $B$ created in VMD. C. BIAR [PDB 2YCW] box plot highlighting the major (purple) and minor (blue) binding pockets from GPCRDB tools (Isberg et al., 2014).

Representation modes that are effective for the extracellular view include both the schematic wheel/box diagrams and several three-dimensional representation modes. The twodimensional schematic wheel/box is effective to depict the contribution of specific amino acid residues in the context of each helix. The wheel/box plot, analogous to the snake plot, provides residue-by-residue information, such as sites of interaction or mutation (see for example Steen et al., 2013, Figure 1). Three-dimensional representation modes that afford visibility of interior structures and reveal relationships between ligands and structural elements effectively include ribbon and rod-cartoon modes, as well as combination modes.

\section{Dynamic Helical Rearrangements Accompanying Activation}

GPCRs respond to ligands by undergoing dynamic conformational changes in the transmembrane domain, which alter the ability of the GPCR to communicate with intracellular signaling partners. These conformational changes in the transmembrane domain are shifts in position and orientation of certain helices within the transmembrane helical bundle. The inactive state is stabilized by the close alignment and interaction of TM3 and TM6 at the intracellular face, a closed conformation that sterically prevents docking of signaling partner proteins (Figure 11). 


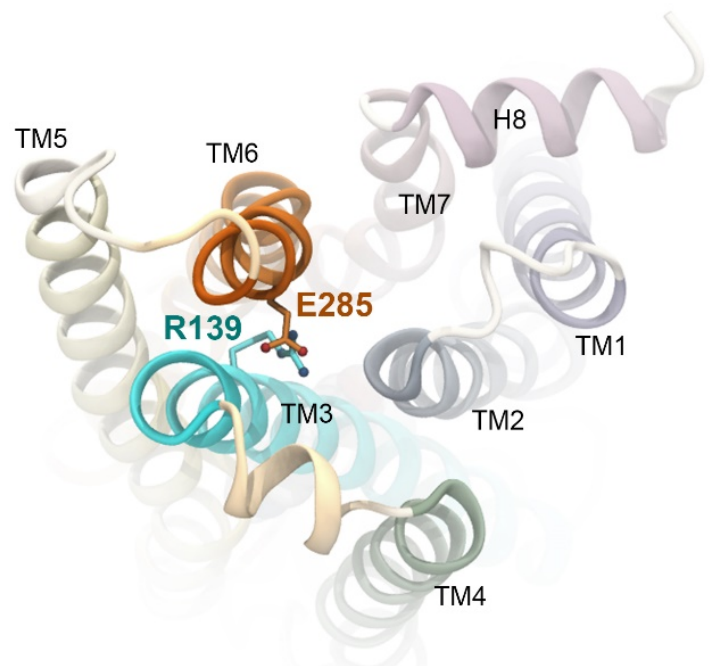

Figure 11. The close alignment of the intracellular tips of TM3 (cyan) and TM6 (orange) in the inactive state allows stabilizing interactions, such as the ionic bond between arginine (R)139 and glutamate (E) 285 in the Turkey $\beta 1$ Adrenergic Receptor [Moukhametzianov et al., 2011 [PDB 2YCW]. Intracellular view. Ribbon representation in combination with stick representation (VMD; NewCartoon/Licorice). Image based upon original illustration created by Faith Simunyu in 2015, with permission.

The activation-related movements of the GPCR can be visualized with a clothespin analogy. The agonist ligand acts as the pressure exerted on the top of the clothespin compressing it and allowing the bottom to open as the GPCR opens on its intracellular face (van der Kant 2014). Upon activation, TM6 swings and twists outwards away from TM3; TM5 moves in coordination with TM6, and TM7 moves and twists inward (Venkatakrishnan et al., 2014), as shown in Figure 12. This opens a crevice on the intracellular face to permit the docking of the intracellular signaling partners such as G proteins and arrestins (Shukla et al., 2014; Katritch et al., 2013). Subsequent intracellular signaling events and regulatory events such as phosphorylation and desensitization, are initiated within or near the intracellular domain as a consequence of these helical shifts and signal protein partner docking (Whalen et al., 2011).
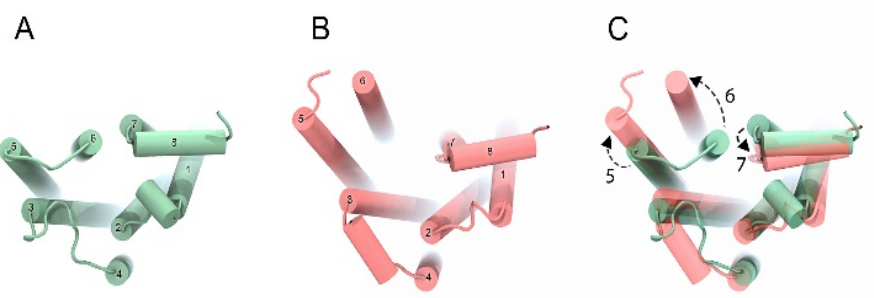

Figure 12. Intracellular view showing dynamic helical shifts upon activation of the $\beta 2$-Adrenergic Receptor (B2AR): Panel A depicts inactive human B2AR [PDB 4GBR], Panel B depicts active human B2AR [PDB 3SN6], Panel C superimposes the same structures shown in panel $A$ and $B$, aligned with Multiseq tool within VMD (Roberts et al., 2006).

\section{Dynamic Helices: Intracellular View}

Dynamic rearrangements at the intracellular face are frequently visualized from an intracellular view. The larger movement of TM6 is apparent in both side and intracellular views, but TM3 may be obscured by intervening structures in the side view that presents TM6 most clearly. Because the relationship between TM3 and TM6 is critical for defining the activation state, a viewpoint which facilitates simultaneous visualization of the position of the intracellular tips of both TM3 and TM6 is useful, and the intracellular view provides this viewpoint (Figures 11 and 12). Additionally, the smaller, subtle movement of TM7 can also be seen from the intracellular view. Ribbon or rod-cartoon representations allow the overall positioning of helices to be appreciated in the intracellular view. The rod-cartoon representation simplifies the helical movements, allowing easy comprehension of the overall choreography of the conformational change. However, a disadvantage of this representation is that the stylized rods underestimate the contribution of the bending/elastic motion of the helices (Katritch et al., 2013).

\section{Conclusions}

This paper strives to provide an entry point to current GPCR science, and to identify visual approaches to communicate select aspects of GPCR structure and function with clarity and accuracy. GPCRs are an important part of human physiology and disease mechanisms, as well as vital therapeutic targets in drug discovery today. The need for accurate GPCR visualizations continues to increase with new discoveries in terms of their structure, function, and conformation flexibility. Medical illustrators can facilitate and promote scientific understanding, contribute to the communication of these discoveries, and enable the success of translational medicine by accurately illustrating GPCRs. To this end, medical illustrators need to understand the basic science to confidently create accurate and visually appealing illustrations, as well as recognizing the pitfalls and potential inaccuracies in their representation. Because GPCRs are of significant research interest to scientists, and continue to yield important discoveries, scientifically accurate visualizations of these receptors are essential for medical illustrators and animators.

\section{Acknowledgements}

This paper is derived from Amy Sojka's project research report, submitted as partial fulfillment of the requirements for the degree of Master of Science, Biomedical Visualization Program, Department of Biomedical and Health Information Sciences, in the Graduate College of the University of Illinois at Chicago, 2014. Amy thanks her research committee Christine Young, Evelyn Maizels, Kevin Brennan, Leah Lebowicz for their aid, guidance, and support in this project. All the authors thank John Daugherty for his leadership and the UIC Biomedical Visualization program for the opportunity to explore and learn about these fascinating proteins. 


\section{References}

Alvarez, B. V., Quon, A. L., Mullen, J., and Casey, J. R. 2013. Quantification of carbonic anhydrase gene expression in ventricle of hypertrophic and failing human heart. $B M C$ Cardiovascular Disorders, 13:2.

Andresen, B. T. 2011. A pharmacological primer of biased agonism. Endocrine, Metabolic \& Immune Disorders Drug Targets, 11(2): 92-98.

Burg, J. S., Ingram, J. R., Venkatakrishnan, A. J., Jude, K. M., Dukkipati, A., Feinberg, E. N., Angelini, A., Waghray, D., Dror, R. O., Ploegh, H. L., and Garcia, K. C. 2015. Structural biology. structural basis for chemokine recognition and activation of a viral G protein-coupled receptor. Science (New York, N.Y.), 347(6226): 1113-1117.

Chabner, B., Brunton L., and Knollman, B. 2011. Goodman and Gilman's The Pharmacological Basis of Therapeutics, Twelfth Edition. New York, NY: McGraw Hill Education.

Costanzi, S., and Wang, K. 2014. The GPCR crystallography boom: Providing an invaluable source of structural information and expanding the scope of homology modeling. Advances in Experimental Medicine and Biology, 796: 3-13.

Couty, J. P., and Gershengorn, M. C. 2005. G-protein-coupled receptors encoded by human herpesviruses. Trends in Pharmacological Sciences, 26(8): 405-411.

Daaka, Y. 2012. S-nitrosylation-regulated GPCR signaling. Biochimica Et Biophysica Acta, 1820(6): 743-751.

Dahmani, H. R., Schneeberger, P., and Kramer, I. M. 2009. Analysis of students' aptitude to provide meaning to images that represent cellular components at the molecular level. CBE Life Sciences Education, 8(3): 226-238.

Dixon, R. A., Kobilka, B. K., Strader, D. J., Benovic, J. L., Dohlman, H. G., Frielle, T., Bolanowski, M. A., Bennett, C. D., Rands, E., Diehl, R. E., Mumford, R. A., Slater, E. E., Sigal, I.S., Caron, M.G., Lefkowitz, R. J., and Strader, C. D. 1986. Cloning of the gene and cDNA for mammalian beta-adrenergic receptor and homology with rhodopsin. Nature, 321(6065): 75-79.

Ferre, S., Casadó, V., Devi, L. A., Filizola, M., Jockers, R., Lohse, M. J., Milligan, G., Pin, J. P., and Guitart, X. 2014. G protein-coupled receptor oligomerization revisited: Functional and pharmacological perspectives. Pharmacological Reviews, 66(2): 413-434.

Fredriksson, R., Lagerstrom, M. C., Lundin, L. G., and Schioth, H. B. 2003. The G-protein-coupled receptors in the human genome form five main families. phylogenetic analysis, paralogon groups, and fingerprints. Molecular Pharmacology, 63(6): 1256-1272.
Goddard, A. D., and Watts, A. 2012. Regulation of G proteincoupled receptors by palmitoylation and cholesterol. $B M C$ Biology, 10: 27.

Goodsell, D. S. 2005. Visual methods from atoms to cells. Structure (London, England: 1993), 13(3): 347-354.

Goodsell, D. S., and Johnson, G. T. 2007. Filling in the gaps: Artistic license in education and outreach. PLoS Biology, 5(12): e308.

Gurevich, V. V., and Gurevich, E. V. 2006. The structural basis of arrestin-mediated regulation of G-protein-coupled receptors. Pharmacology \& Therapeutics, 110(3): 465-502.

Hargrave, P. A., McDowell, J. H., Curtis, D. R., Wang, J. K., Juszczak, E., Fong, S. L., Rao, J. K., and Argos, P. 1983. The structure of bovine rhodopsin. Biophysics of Structure and Mechanism, 9(4): 235-244.

Harle, M., and Towns, M. H. 2012a. Students' understanding of external representations of the potassium ion channel protein, part I: Affordances and limitations of ribbon diagrams, vines, and hydrophobic/polar representations. Biochemistry and Molecular Biology Education, 40(6): 349-356.

Harle, M., and Towns, M. H. 2012b. Students' understanding of external representations of the potassium ion channel protein part II: Structure-function relationships and fragmented knowledge. Biochemistry and Molecular Biology Education, 40(6): 357-363.

Humphrey, W., Dalke, A. and Schulten, K. 1996. VMD - Visual Molecular Dynamics. Journal of Molecular Graphics, 14(1): 3338.

Innamorati, G., Valenti, M.T., Giovinazzo, F., Carbonare, L. D., Parenti, M., and Bassi, C. 2011. Molecular Approaches To Target GPCRs in Cancer Therapy. Pharmaceuticals, 4(4): 567-589.

Isberg, V., Vroling, B., van der Kant, R., Li, K., Vriend, G., and Gloriam, D. 2014. GPCRDB: An information system for G protein-coupled receptors. Nucleic Acids Research, 42(D1): D422-5.

Jenkinson, J., and McGill, G. 2013. Using 3D Animation in Biology Education: Examining the Effects of Visual Complexity in the Representation of Dynamic Molecular Events. Journal of Biocommunication, 39(2):42-49.

Katritch, V., Cherezov, V., and Stevens, R. C. 2012. Diversity and modularity of G protein-coupled receptor structures. Trends in Pharmacological Sciences, 33(1): 17-27.

Katritch, V., Cherezov, V., and Stevens, R. C. 2013. Structurefunction of the G protein-coupled receptor superfamily. Annual Review of Pharmacology and Toxicology, 53: 531-556. 
Kenakin, T. 2011. Functional selectivity and biased receptor signaling. The Journal of Pharmacology and Experimental Therapeutics, 336(2): 296-302.

Kobilka, B. K., Dixon, R. A., Frielle, T., Dohlman, H. G., Bolanowski, M. A., Sigal, I. S., Yang-Feng, T. L., Francke, U., Caron, M. G.,and Lefkowitz, R. J. 1987. cDNA for the human beta 2-adrenergic receptor: A protein with multiple membranespanning domains and encoded by a gene whose chromosomal location is shared with that of the receptor for platelet-derived growth factor. Proceedings of the National Academy of Sciences of the United States of America, 84(1): 46-50.

Kramer, I. M., Dahmani, H. R., Delouche, P., Bidabe, M., and Schneeberger, P. 2012. Education catching up with science: Preparing students for three-dimensional literacy in cell biology. CBE Life Sciences Education, 11(4): 437-447.

Krumm, B. E., and Grisshammer, R. 2015. Peptide ligand recognition by $\mathrm{G}$ protein-coupled receptors. Frontiers in Pharmacology, 6: 48.

Langer, I. 2012. Mechanisms involved in VPAC receptors activation and regulation: Lessons from pharmacological and mutagenesis studies. Frontiers in Endocrinology, 3: 129.

Lee, S. M., Booe, J. M., and Pioszak, A. A. 2015. Structural insights into ligand recognition and selectivity for classes A, B, and C GPCRs. European Journal of Pharmacology, 763(Pt B):196-205.

Lefkowitz, R. J. 2013. A brief history of G-protein coupled receptors (nobel lecture). Angewandte Chemie (International Ed.in English), 52(25): 6366-6378.

Loertscher, J., Villafane, S. M., Lewis, J. E., and Minderhout, V. 2014. Probing and improving student's understanding of protein alpha-helix structure using targeted assessment and classroom interventions in collaboration with a faculty community of practice. Biochemistry and Molecular Biology Education, 42(3): 213-223.

Lomize, M. A., Pogozheva, I. D., Joo, H., Mosberg, H. I., and Lomize, A. L. 2012. OPM database and PPM web server: Resources for positioning of proteins in membranes. Nucleic Acids Research, 40(Database issue): D370-6.

Manglik, A., Kruse, A. C., Kobilka, T. S., Thian, F. S., Mathiesen, J. M., Sunahara, R. K., Pardo, L., Weis, W. I., Kobilka, B. K. and Granier, S. 2012. Crystal structure of the micro-opioid receptor bound to a morphinan antagonist. Nature, 485(7398): 321-326.

Millar, R. P., and Newton, C. L. 2010. The year in G proteincoupled receptor research. Molecular Endocrinology (Baltimore, Md.), 24(1): 261-274.
Moreira, I. S. 2014. Structural features of the G-protein/GPCR interactions. Biochimica Et Biophysica Acta, 1840(1): 16-33.

Moukhametzianov, R., Warne, T., Edwards, P. C., Serrano-Vega, M. J., Leslie, A. G., Tate, C. G., and Schertler, G. F. 2011. Two distinct conformations of helix 6 observed in antagonist-bound structures of a beta1-adrenergic receptor. Proceedings of the National Academy of Sciences of the United States of America, 108(20): 8228-8232.

Mura, C., McCrimmon, C. M., Vertrees, J., and Sawaya, M. R. 2010. An introduction to biomolecular graphics. PLoS Computational Biology, 6(8): pii: e1000918.

O'Donoghue, S. I., Goodsell, D. S., Frangakis, A. S., Jossinet, F., Laskowski, R. A., Nilges, M., Saibil, H. R., Schafferhans, A., Wade, R. C., Westhof, E. and Olson, A. J. 2010. Visualization of macromolecular structures. Nature Methods, 7(3 Suppl): S42-S55.

Palczewski, K., Kumasaka, T., Hori, T., Behnke, C. A., Motoshima, H., Fox, B. A., Le Trong, I., Teller, D. C., Okada, T., Stenkamp, R. E., Yamamoto, M., and Miyano, M. 2000. Crystal structure of rhodopsin: A G protein-coupled receptor. Science (New York, N.Y.), 289(5480): 739-745.

Park, S. H., Das, B. B., Casagrande, F., Tian, Y., Nothnagel, H. J., Chu, M., Kiefer, H., Maier, K., De Angelis, A. A., Marassi, F. M. and Opella, S. J. 2012. Structure of the chemokine receptor CXCR1 in phospholipid bilayers. Nature, 491(7426): 779-783.

Perkins, J. A. 2005a. A History of Molecular Representation Part 1: 1800 to the 1960s. Journal of Biocommunication, 31(1): 1-13.

Perkins, J. A. 2005b. A History of Molecular Representation Part 2: the 1960s to Present. Journal of Biocommunication, 31(2): 1-14.

Pettersen, E. F., Goddard, T. D., Huang, C. C., Couch, G. S., Greenblatt, D. M., Meng, E. C., and Ferrin, T. E. 2004. UCSF Chimera - a visualization system for exploratory research and analysis. Journal of Computational Chemistry, 25(13): 1605-1612.

Piscitelli, C. L., Kean, J., de Graaf, C., and Deupi, X. 2015. A molecular pharmacologist's guide to $\mathrm{G}$ protein-coupled receptor crystallography. Molecular Pharmacology, 88(3): 536-551.

Rasmussen, S. G., DeVree, B. T., Zou, Y., Kruse, A. C., Chung, K. Y., Kobilka, T. S., Thian, F. S., Chae, P. S., Pardon, E., Calinski, D., Mathiesen, J. M., Shah, S. T., Lyons, J. A., Caffrey, M., Gellman, S. H., Steyaert, J., Skiniotis, G., Weis, W. I., Sunahara, R. K., and Kobilka, B. K. 2011. Crystal structure of the ß2 adrenergic receptor-gs protein complex. Nature, 477(7366): 549-555.

Richardson, J. S., and Richardson, D. C. 2013. Doing molecular biophysics: Finding, naming, and picturing signal within complexity. Annual Review of Biophysics, 42:1-28. 
Richardson, J. S., Richardson, D. C., Tweedy, N. B., Gernert, K. M., Quinn, T. P., Hecht, M. H., Erickson, B. W., Yan, Y., McClain, R. D., and Donlan, M. E. 1992. Looking at proteins: Representations, folding, packing, and design. biophysical society national lecture, 1992. Biophysical Journal, 63(5): 11851209.

Roberts, E., Eargle, J., Wright, D., and Luthey-Schulten, Z. 2006. MultiSeq: Unifying sequence and structure data for evolutionary analysis. BMC Bioinformatics, 7:382.

Rosenkilde, M. M., Benned-Jensen, T., Frimurer, T. M., and Schwartz, T. W. 2010. The minor binding pocket: A major player in 7TM receptor activation. Trends in Pharmacological Sciences, 31(12): 567-574.

Schaffhausen, J. 2014. Towards understanding bias at GPCRs. Trends in Pharmacological Sciences, 35(6): 267.

Shibata, Y., White, J. F., Serrano-Vega, M. J., Magnani, F., Aloia, A. L., Grisshammer, R., and Tate, C. G. 2009. Thermostabilization of the neurotensin receptor NTS1. Journal of Molecular Biology, 390(2): 262-277.

Shukla, A. K., Westfield, G. H., Xiao, K., Reis, R. I., Huang, L. Y., Tripathi-Shukla, P., Qian, J., Li, S., Blanc, A., Oleskie, A. N., Dosey, A. M., Su, M., Liang, C. R., Gu. L. L., Shan, J. M., Chen, X., Hanna, R., Choi, M., Yao, X. J., Klink, B. U., Kahsai, A. W., Sidhu, S. S., Koide, S., Penczek, P. A., Kossiakoff, A. A., Woods, V. L.Jr, Kobilka, B. K., Skiniotis, G., and Lefkowitz, R. J. 2014. Visualization of arrestin recruitment by a G-proteincoupled receptor. Nature, 512(7513): 218-222.

Siu, F. Y., and Stevens, R. C. 2010. RAMP-ing up class-B GPCR ECD structural coverage. Structure (London, England: 1993), 18(9): 1067-1068.

Smith, N. J., and Milligan, G. 2010. Allostery at G proteincoupled receptor homo- and heteromers: Uncharted pharmacological landscapes. Pharmacological Reviews, 62(4): 701-725.

Sodhi, A., Montaner, S., and Gutlind, J. S. 2004. Viral hijacking of $\mathrm{G}$ protein-coupled receptor signaling networks. Nature Reviews Molecular Cell Biology, 5(12): 998-1012.

Standfuss, J., Edwards, P. C., D'Antona, A., Fransen, M., Xie, G., Oprian, D. D., and Schertler, G. F. 2011. The structural basis of agonist-induced activation in constitutively active rhodopsin. Nature, 471(7340): 656-660.

Steen, A., Thiele, S., Guo, D., Hansen, L. S., Frimurer, T. M., and Rosenkilde, M. M. 2013. Biased and constitutive signaling in the $\mathrm{CC}$-chemokine receptor CCR5 by manipulating the interface between transmembrane helices 6 and 7. The Journal of Biological Chemistry, 288(18): 12511-12521.

van der Kant, R., and Vriend, G. 2014. Alpha-bulges in G protein-coupled receptors. International Journal of Molecular Sciences, 15(5): 7841-7864.

Venkatakrishnan, A. J., Flock, T., Prado, D. E., Oates, M. E., Gough, J., and Madan Babu, M. 2014. Structured and disordered facets of the GPCR fold. Current Opinion in Structural Biology, 27: $129-137$.

Villafane, S.M., Loertscher, J., Minderhout V., and Lewis J. E. 2011. Uncovering students' incorrect ideas about foundational concepts for biochemistry. Chemistry Education Research and Practice, 12(2): 210-218.

Violin, J. D., and Lefkowitz, R. J. 2007. Beta-arrestin-biased ligands at seven-transmembrane receptors. Trends in Pharmacological Sciences, 28(8): 416-422.

Vischer, H. F., Watts, A. O., Nijmeijer, S., and Leurs, R. 2011. G protein-coupled receptors: Walking hand-in-hand, talking hand-inhand? British Journal of Pharmacology, 163(2): 246-260.

von Heijne, G. 2006. Membrane-protein topology. Nature Reviews.Molecular Cell Biology, 7(12): 909-918.

Warne, T., Edwards, P. C., Leslie, A. G., and Tate, C. G. 2012. Crystal structures of a stabilized beta1-adrenoceptor bound to the biased agonists bucindolol and carvedilol. Structure (London, England: 1993), 20(5): 841-849.

Whalen, E. J., Rajagopal, S., and Lefkowitz, R. J. 2011. Therapeutic potential of beta-arrestin- and $\mathrm{G}$ protein-biased agonists. Trends in Molecular Medicine, 17(3): 126-139.

Wong, B. 2010. Salience. Nature Methods, 7(10): 773.

Wong, B. 2011a. Salience to relevance. Nature Methods, 8(11): 889.

Wong, B. 2011b. Simplify to clarify. Nature Methods, 8(8): 611.

\section{Appendix - Additional Topics of Interest}

\section{GPCRs Coupling to Binding Partners}

Activated GPCRs interact with a variety of heterotrimeric Gproteins composed of alpha, beta, and gamma subunits. Interaction of the alpha subunit with the GPCRs causes the alpha subunit to release GDP and bind GTP. This exchange causes the alpha protein to dissociate from the beta/gamma heterodimer. The G protein subunits then interact with effector proteins causing downstream signaling cascades (Moreira, 2014).

Arrestins are a small family of proteins. They activate or redirect pathways that regulate signal transduction. Arrestins signal in a positive manner through activation of downstream kinase cascades. They also play a role in receptor desensitization and internalization (Violin \& Lefkowitz, 2007). 


\section{GPCR Specialty Features}

GPCRs have several specialty features that contribute to their importance in the pharmaceutical world. Medical illustrators should understand these features because it is likely that they will be called upon to depict them due to their importance to the GPCRs unique functionality.

\section{Functional Selectivity}

Functional selectivity or biased agonists are an aspect of ligand binding. It is a feature that allows a GPCR to only act upon a subset of its activation pathways. Pharmaceuticals could target the biased agonists to fine tune the receptor for a specific function. More information is available through (Andresen, 2011); (Kenakin, 2011).

\section{Allosteric and Orthosteric Relationships}

Allosteric "allo-" means "other," and its most general definition is: interaction between two or more topographically distinct sites. Orthosteric ligand binding sites are considered the natural binding sites while allosteric ligand binding sites are located in many different places in the GPCR. The allosteric ligand can alter the potency of the orthosteric ligand as well as the overall GPCR function. For more information visit (Smith \& Milligan, 2010).

\section{Oligomerization}

GPCRs are often described as monomers, but they can interact and form dimers and larger oligomers. These dimeric or oligomeric associations can either be permanently stable or partners may work together once and disassociate. The consequences of complex formation for signaling are still an area of active research. More information is available in (Ferre et al., 2014).

\section{Authors}

Amy C. Sojka, M.S. is the senior web designer/medical illustrator at Medline Industries Inc. She received her Masters of Science from the University of Illinois at Chicago Biomedical Visualization program in 2014. It was during her time in the BVIS program that Amy developed an interest GPCRs. Ms. Sojka can be contacted at ac.sojka@gmail.com

Kevin Brennan, M.S., CMI is a Clinical Assistant Professor in the Biomedical Visualization Program at the University of Illinois of Chicago. Prior to coming to UIC he worked at Argosy Publishing as a medical animator and as senior content developer for the award winning Visible Body. He is the head and founder of BioViz Studios. He earned his BA in Biology from Illinois Wesleyan University in 2003 and his MS in Biomedical Visualization from the University of Illinois at Chicago in 2005.
Evelyn Maizels M.D., Ph.D., M.S. in Biomedical Visualization. Following a first career in biomedical research focusing on signal transduction as a member of the research faculty of the Department of Cell and Molecular Biology at Northwestern University's Feinberg School of Medicine, Evelyn completed the Masters in the Biomedical Visualization program at the University of Illinois at Chicago in 2010. Her course of study at UIC has allowed her to merge her interest in art with her scientific background, and introduced her to many new concepts, practices and technologies. She works together with her husband to create e-learning at CEVL (Computer Enhanced Visual Learning) and enjoys teaching molecular pharmacology to UIC students.

Christine Young, M.A., CMI, FAMI is a Clinical Assistant Professor in the Biomedical Visualization Graduate Program at University of Illinois at Chicago and owns a small studio in Evanston, IL dedicated to life science visualization.

\section{Licensing}

The authors have chosen to license this content under a Creative Commons Attribution, NonCommercial, NoDerivatives License 4.0 International License.

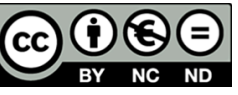

